\title{
Development of a Preliminary Ultrasonographic Enthesitis Score in Psoriatic Arthritis - GRAPPA Ultrasound Working Group
}

\author{
Stephanie Tom, Yujie Zhong, Richard Cook, Sibel Zehra Aydin, Gurjit Kaeley, and Lihi Eder
}

\begin{abstract}
Objective. To assess the performance of various sonographic elemental entheseal lesions in distinguishing between psoriatic arthritis (PsA) and controls to inform the development of a novel sonographic enthesitis score for PsA.

Methods. A total of 100 age- and sex-matched individuals (50 PsA and 50 controls) were evaluated. Eleven entheseal sites were scanned bilaterally according to a standardized protocol by 2 sonographers. Based on the Outcome Measures in Rheumatology (OMERACT) definition of sonographic enthesitis, the following lesions were assessed: structural entheseal changes (hypoechogenicity), thickening, bone erosion, enthesophytes, calcification, and Doppler signal, in addition to bursitis and bone irregularities. The images were read by 2 readers blinded to the clinical information. A series of logistic regression models were used to find the optimal combination of entheseal sites and elementary lesions that distinguished PsA from controls.

Results. Mean age was $55 \pm 10$ years (59\% males). The optimal model that distinguished PsA from controls included 5 elementary lesions (enthesophytes, Doppler signal, erosions, thickening, and hypoechogenicity) and 6 entheseal sites (patellar ligament insertions into the distal patella and tibial tuberosity, Achilles tendon and plantar fascia insertions into the calcaneus, common extensor tendon insertion into lateral epicondyle, and supraspinatus insertion into the superior facet of the humerus). The area under the receiver-operating characteristic curve for this model was 0.93 (95\% CI 0.88-0.98). Conclusion. We identified potential elemental ultrasonographic abnormalities and entheseal sites that could distinguish PsA and controls. This information will contribute to the development of a new sonographic score for assessment of enthesitis in patients with PsA. (First Release October 15 2018; J Rheumatol 2019;46:384-90; doi:10.3899/jrheum.171465)
\end{abstract}

Key Indexing Terms:

PSORIATIC ARTHRITIS ULTRASONOGRAPHY OUTCOMES DIAGNOSTIC IMAGING

From the Division of Rheumatology, Toronto Western Hospital, Toronto; Division of Rheumatology, Women's College Hospital, Toronto; Division of Rheumatology, Department of Medicine, University of Toronto, Toronto; University of Waterloo, Waterloo, Ontario; Division of Rheumatology, Ottawa Hospital, University of Ottawa, Ottawa Hospital Research Institute, Ottawa, Ontario, Canada; MRC Biostatistics Unit, School of Clinical Medicine, University of Cambridge, Cambridge, UK; Division of Rheumatology, University of Florida, Jacksonville, Florida, USA.

Dr. Tom was supported by a Research Scholarship from the Canadian Rheumatology Ultrasound Society (CRUS). Dr. Eder is supported by a New Investigator Salary Grant from the Arthritis Society and the Canadian Association of Psoriasis Patients.

S. Tom, MD, Division of Rheumatology, Toronto Western Hospital, and Division of Rheumatology, Women's College Hospital, and Division of Rheumatology, Department of Medicine, University of Toronto; Y. Zhong, PhD, MRC Biostatistics Unit, School of Clinical Medicine, University of Cambridge; R. Cook, PhD, University of Waterloo; S.Z. Aydin, MD, Division of Rheumatology, Ottawa Hospital, University of Ottawa, Ottawa Hospital Research Institute; G. Kaeley, MD, Division of Rheumatology, University of Florida; L. Eder, $M D, P h D$, Division of Rheumatology, Women's College Hospital, and Division of Rheumatology, Department of Medicine, University of Toronto.

Address correspondence to Dr. L. Eder, Women's College Research Institute, Room 6326, Women's College Hospital, 76 Grenville St., Toronto, Ontario M5S 1B2, Canada.E-mail: lihi.eder@wchospital.ca

Accepted for publication July 18, 2018.
Enthesitis is a key feature in psoriatic arthritis (PsA). About one-third of patients with PsA develop clinical enthesitis at some point during the course of their disease and its severity is associated with worse disease outcomes ${ }^{1,2}$. Enthesitis may be the initial site of musculoskeletal inflammation in PsA and explains many of the clinical features of the disease ${ }^{3,4}$. Therefore, assessment of enthesitis has been recommended in every patient with PsA 5 .

However, the accuracy of clinical examination of enthesitis is limited to imaging ${ }^{6,7,8}$. Numerous studies have used ultrasound (US) to evaluate various entheseal lesions in patients with spondyloarthritis ( $\mathrm{SpA}$ ) and PsA for diagnostic purposes, to monitor treatment response, and for research of disease pathogenesis ${ }^{9}$. Several global enthesitis scoring methods have been developed to quantify the extent of enthesitis at the patient level such as the Madrid Sonographic Enthesitis Index (MASEI) ${ }^{10}$ and Glasgow Ultrasound Enthesitis Scoring System 7 ; other studies used ad hoc scoring methods or modifications of existing methods to evaluate enthesitis 9 . This lack of consistency led to difficulties in comparing results across studies. 
The Outcome Measures in Rheumatology (OMERACT) US special interest group reached a consensus regarding the sonographic elementary lesions defining SpA-related enthesitis ${ }^{11}$. This was an important first step toward ensuring a high degree of consistency across studies. However, while this group defined the concept of enthesitis at the level of the enthesis, it did not address the issue of evaluating the extent of enthesitis at the global patient level, identify the entheseal sites of specific interest for inflammatory disease, or explore the combination of which lesions are indicative of active enthesitis versus irreversible entheseal damage.

Preliminary work of the Group for Assessment of Psoriasis and Psoriatic Arthritis (GRAPPA) US working group highlighted major gaps in knowledge regarding the validity of existing sonographic enthesitis scoring systems in $\mathrm{PsA}^{12}$. In our systematic literature review, the vast majority of existing methods were developed in patients with predominantly axial SpA, and the validity of those methods in PsA is unknown. There is limited information about the discriminative validity and responsiveness of existing scoring systems in PsA. Additional limitations include the predominance of lower extremities sites, expert opinion-driven development process and limited data about the effect of confounding factors such as age, sex, and weight on the various scores.

Owing to these notable limitations, the GRAPPA US working group has set up a goal to develop and validate a novel enthesitis sonographic score for PsA through a multistep process, using a combined data-driven and expert opinion-driven approach. The objective of this preliminary study was to assess the performance of various sonographic entheseal lesions and sites in distinguishing between PsA and healthy controls. This will inform the development of a novel sonographic enthesitis score for PsA.

\section{MATERIALS AND METHODS}

Patients and setting. A total of 50 patients with PsA and 50 healthy controls who were roughly matched by age and sex were recruited for this crosssectional study from April 2016 to March 2017. Patients with PsA from 2 academic medical centers in Canada were enrolled for a single US assessment. All patients satisfied the Classification for Psoriatic Arthritis criteria $^{13}$. We aimed to include a wide spectrum of disease manifestations, severities, and durations. Therefore, no predefined strict inclusion criteria were set. We enrolled patients with active PsA who had at least 1 tender entheseal site or an actively inflamed peripheral joint. Healthy controls who had no personal or family history of inflammatory arthritis or psoriasis were recruited from hospital personnel and through advertisements. This study was approved by the University Health Network and Women's College Hospital Research Ethics Boards (approval 2013-0052E). All participants signed an informed consent form.

Clinical data collected. The collected information included demographics and body mass index (BMI) for all participants. Examination of clinical enthesitis according to the Spondyloarthritis Research Consortium Canada index was performed in patients and controls by a rheumatologist independently of the sonographic data.

US protocol: entheseal sites. The selection of entheseal sites for previous scoring systems was primarily based on expert opinion, which may have led to omission of important entheseal sites. Therefore, in our present study, we aimed to scan a wide range of entheseal sites in the upper and lower extremities. The selection of entheseal sites was conducted in a multistep process that included identification of potentially useful entheseal sites for scanning based on literature review, existing scores for clinical and sonographic enthesitis, and expert opinion of rheumatologists experienced in musculoskeletal US in PsA (LE, GK, SA). We identified 14 entheseal sites that were evaluated in a small pilot study that included 11 patients with PsA. Because of technical difficulties in visualizing the entheses and distinguishing normal and abnormal lesions, we excluded 3 sites (sartorius insertion onto anterior superior iliac spine, medial collateral ligament at femoral insertion, and peroneal tendon adjacent to lateral malleolus as functional entheses) from the study protocol. Thus, the final protocol included the following 11 anatomic sites for bilateral scanning (total scanning of 22 entheseal sites): quadriceps insertion at superior patella, patellar ligament insertions at the distal patella and tibial tuberosity, tibialis posterior next to medial malleolus as a functional entheseal site, Achilles tendon insertion into calcaneus, plantar fascia insertion into calcaneus, triceps tendon insertion into olecranon tuberosity, common extensor tendon at lateral epicondyle, common flexor tendon at medial epicondyle, supraspinatus insertion into superior facet of greater tubercle of humerus, and deltoid insertion into deltoid tuberosity of the humerus.

Elementary lesions of enthesitis. The OMERACT consensus manuscript from 2014 was used as the basis for defining sonographic elementary lesions ${ }^{11}$. We further added to the protocol several lesions of interest that were thought to represent inflammatory enthesitis and were not included in the consensus statement. Some of the lesions were scored as binary outcomes (absent/present) and others were graded based on their severity using a semiquantitative scale of 0 to 3 . We provide an illustrated atlas that includes detailed definitions and examples of sonographic elementary lesions evaluated in this study (Supplementary Data 1, available with the online version of this article).

The following 8 sonographic entheseal lesions were evaluated:

(1) Structural changes (hypoechogenicity), defined as loss of homogeneous fibrillar pattern of the tendon/ligament at the enthesis not attributed to anisotropy (score: $0=$ absent, $1=$ present).

(2) Thickening, defined as increased thickness of the tendon/ligament at the enthesis compared to its body based on a vertical measurement in longitudinal axis starting from cortical bone of entheseal attachment at the widest section of enthesis. Cutoff points were based on previously published studies ${ }^{10}$. Thickening was evaluated only for the 6 entheseal sites that are included in the MASEI score and the lateral epicondyle because no cutoff points are available for other entheseal sites (supraspinatus, tibialis posterior, common extensor and flexor, deltoid; score: $0=$ absent, $1=$ present).

(3) Bone erosion, defined as cortical break confirmed with a stepdown contour defect detected in 2 planes at the insertion of the tendon/ligament to the bone (score: $0=$ absent, 1 = present).

(4) Bone irregularities, defined as "fluffy" hyperechoic bone projections originating from the bone along the enthesis (score: $0=$ absent, $1=$ present).

(5) Enthesophyte, defined as a step-up bony prominence at the normal bone contour. Enthesophytes were further graded by anatomic location (traction vs nontraction) and size. Traction enthesophyte was defined as a single enthesophyte originating at the end of the attachment of tendon/ligament to bone. Nontraction enthesophyte was defined as enthesophyte(s) located along the attachment of the tendon/ligament to the bone. We used a semiquantitative scale to grade the enthesophytes based on their size (score: 0 = absent, $1=$ small, 2 = medium, 3 = large $)$.

(6) Calcifications, defined as hyperechoic linear structures detected within the tendon/ligament at the attachment to the bone but with no congruency with the bone (score: 0 = absent, 1 = present).

(7) Bursitis, defined as hypoechoic area located within known anatomic bursa that is displaceable with pressure (score: $0=$ absent, $1=$ present).

(8) Doppler signal, defined as the presence of positive Doppler signal at the enthesis, confirmed in 2 perpendicular planes, and distinguished from

Personal non-commercial use only. The Journal of Rheumatology Copyright (c) 2019. All rights reserved. 
reflection surface artifacts and nutritional vessel signal. Doppler signal was further classified based on location and intensity. According to the OMERACT definition, positive Doppler signal should be found within $2 \mathrm{~mm}$ of the bony cortex. However, this is an arbitrary cutoff because the histologic boundaries of the enthesis are not well defined. Further, according to the synovio-entheseal complex model, the bursa is considered an integral part of the enthesis; thus, Doppler signal at the bursa may represent active inflammatory enthesitis. Therefore, we extended the areas evaluated for the presence of Doppler signal and defined 3 anatomical zones: (1) within $2 \mathrm{~mm}$ of the cortical bone; (2) within 2-5 mm of the cortical bone; and (3) within the adjacent bursa. In addition, the intensity of Doppler signal was graded on a semiquantitative scale of $0-3$ : $0=$ absent; $1=$ mild; $2=$ moderate; $3=$ severe.

Sonographic evaluation. All individuals were scanned by one of 2 rheumatologists (LE, ST). The senior investigator (LE) had 7 years of experience in musculoskeletal US with a particular interest in enthesitis in patients with PsA. The second sonographer (ST) had 3 years of experience in musculoskeletal US and was trained and supervised by the senior investigator. MyLab 70 XVG (Esaote) and MyLab Twice (Esaote) scanners equipped with a 6-18 MHz linear array transducer (Esaote) were the US devices. Power Doppler settings were standardized with a Doppler frequency of 8.3-10 MHz (depending on body habitus), pulse repetition frequency of $750 \mathrm{~Hz}$, and a wall filter of 2

Eleven entheseal sites were assessed bilaterally according to the protocol described above. Each enthesis was scanned in both longitudinal and transverse planes. Each examination took about 30-45 min. The patients were placed in a supine position to assess the patellar and quadriceps entheses. The knee was placed in $30^{\circ}$ flexion to assess greyscale abnormalities and in full extension to assess vascularization. The patients were then placed in a sitting position with the feet dangling for assessment of the Achilles tendon, tibialis posterior, and plantar fascia entheses. The triceps tendon and common extensor tendon entheses were assessed with the elbow flexed to $90^{\circ}$. The common flexor tendon enthesis was evaluated with the elbow fully extended. The supraspinatus attachment was scanned in modified Crass position (internal rotation of the shoulder with the palm placed on the superior aspect of the iliac wing), and the deltoid attachment to the lateral acromion was scanned in resting neutral arm.

Since blinding for the clinical diagnosis is difficult owing to the occasional presence of skin psoriasis over entheseal sites, each scan was recorded and stored as a short video file for later reading. Images were read and scored independently by 2 readers (LE, ST) who were blinded to the clinical information including the patient diagnosis. Intra- and interobserver reliability were assessed by reading 220 images of study participants at 2 different timepoints more than 1 month apart. The intra- and interrater reliability for scoring all lesions were excellent (ICC 0.92-0.96).

Statistical analysis. Descriptive statistics used with continuous data were described by mean \pm SD and categorical variables were expressed as frequencies and percentages. Chi-square tests or Fisher's exact test were used to compare categorical variables, and t test was used for continuous variables.

The frequency of each elementary lesion was tabulated and compared between patients with PsA and controls, and the sensitivity and specificity of the presence of these lesions to identify PsA were computed.

To identify the optimal combination of information on entheseal sites and lesions that discriminates between PsA and controls, we performed a 3 -step process that involved performing logistic regression analysis and backward elimination. Our first aim was to narrow down the number of elementary lesions. Therefore, we fitted a series of individual multivariable logistic regression models for each of the elementary lesions evaluated in the study. For each elementary lesion, the model covariates included the score for that lesion in each of the 11 entheseal sites and the model outcome was disease status (PsA vs control). Elementary lesions that were associated with disease status in at least 1 entheseal site (significance level p < 0.10) after backward elimination were selected to be analyzed in the next phase. In the second phase, we fitted a logistic regression model for each entheseal site with only elementary lesions identified in the first phase as covariates. We then used the estimated regression coefficients to construct risk scores for each of the 11 entheseal sites. In the last step, we performed a logistic regression model with the risk scores obtained from the second phase as predictors of disease status. We used backward elimination to identify the significant entheseal sites associated with PsA $(\mathrm{p}<0.10)$. We reported the area under the receiver-operating characteristic curve (AUC) for the final regression models.

\section{RESULTS}

A total of 50 patients with PsA and 50 healthy controls were included in the analysis. The characteristics of the study population are shown in Table 1. Patients and controls were similar regarding their mean age and sex distribution. As expected, BMI was higher in patients with PsA (PsA: $28.9 \pm$ SD $4.8 \mathrm{~kg} / \mathrm{m}^{2}$ vs controls: $24.7 \pm 3.8 \mathrm{~kg} / \mathrm{m}^{2}$ ). The mean duration of PsA was $14.6 \pm 12.3$ years and the prevalence of at least 1 tender entheseal site was $42 \%$ in PsA and $22 \%$ in the controls.

Frequencies of sonographic entheseal lesions. The frequencies of the various elementary lesions and their sensitivity and specificity for PsA are shown in Table 2 (detailed frequencies of each lesion by site are provided in Supplementary Tables $2 \mathrm{~A}-\mathrm{N}$, available with the online version of this article). The most frequent elementary lesions were any enthesophytes (grade $>0$ ), thickening, and hypoechogenicity, which were found in up to one-third of the entheses in patients with PsA. However, these lesions were also quite frequent in healthy individuals, observed in up to one-quarter of the individuals, resulting in lower specificities (specificities for enthesophyte, thickening, and hypoechogenicity were $0.76,0.84$, and 0.90 , respectively). In contrast, other elementary lesions including Doppler signal, bone proliferation, and erosions were rarely found in healthy controls. The frequency of these lesions was 5.4 to 6.9 times higher in the entheses of patients with PsA compared with controls; however, their prevalence even in patients with PsA was relatively low (range 5.1-12.5\%), which resulted in lower sensitivity rates. The frequency of bursitis, nontraction enthesophytes, and calcifications was similar in patients and controls.

Table 1. Characteristics of the study participants.

\begin{tabular}{lccc}
\hline Characteristics & PsA & Controls & $\mathrm{p}$ \\
\hline Age, yrs & $55 \pm 12.2$ & $55.1 \pm 8.3$ & 0.96 \\
Sex, female & $20(40.0)$ & $21(42.0)$ & 0.84 \\
BMI, kg/m² & $28.9 \pm 4.8$ & $24.7 \pm 3.8$ & $<0.001$ \\
Psoriasis duration, yrs & $24.7(16.1)$ & - & \\
PsA duration, yrs & $14.6(12.3)$ & - & \\
Clinical enthesitis $(\geq 1$ tender & & $11(22)$ & 0.03 \\
$\quad$ entheseal site) & $21(42)$ & $0.6 \pm 1.5$ & 0.02 \\
Tender entheseal count & $1.7 \pm 2.9$ & & \\
\hline
\end{tabular}

Data are expressed as $\mathrm{n}(\%)$ or mean $\pm \mathrm{SD}$. PsA: psoriatic arthritis; BMI: body mass index. 
Table 2. Frequency of sonographic entheseal lesions (per entheseal sites).

\begin{tabular}{|c|c|c|c|c|c|}
\hline Variables & PsA, n $(\%)$ & Controls, $\mathrm{n}(\%)$ & $\mathrm{p}$ & Sensitivity & Specificity \\
\hline Structural changes & $114(20.7)$ & $41(7.5)$ & $<0.001$ & 0.21 & 0.93 \\
\hline Thickening & $97(32.3)$ & $48(16)$ & $<0.001$ & 0.32 & 0.84 \\
\hline Enthesophyte, any type (grade $>0$ ) & $195(35.5)$ & $135(24.6)$ & $<0.001$ & 0.36 & 0.76 \\
\hline Enthesophyte, any type (grade $>1$ ) & $102(18.5)$ & $53(9.7)$ & $<0.001$ & 0.19 & 0.90 \\
\hline Enthesophyte, any type (grade $>2$ ) & $50(9.1)$ & $27(4.9)$ & 0.009 & 0.09 & 0.95 \\
\hline Erosions & $69(12.5)$ & $10(1.8)$ & $<0.001$ & 0.13 & 0.98 \\
\hline Bone irregularities & $28(5.1)$ & $5(0.9)$ & $<0.001$ & 0.05 & 0.99 \\
\hline Doppler $($ grade $>0)$ & $77(14)$ & $14(2.6)$ & $<0.001$ & 0.14 & 0.98 \\
\hline Doppler $($ grade $>1)$ & $37(6.7)$ & $2(0.4)$ & $<0.001$ & 0.07 & 0.99 \\
\hline Doppler $($ grade $>2)$ & $9(1.6)$ & $1(0.2)$ & 0.03 & 0.02 & 0.99 \\
\hline Doppler involving zone 1 (grade $>0)$ & $65(11.8)$ & $11(2)$ & $<0.001$ & 0.12 & 0.98 \\
\hline
\end{tabular}

PsA: psoriatic arthritis.

Identification of key elementary lesions distinguishing PsA from controls. In a series of multivariable regression models fitted for each elementary lesion separately, we aimed to narrow down the number of elementary sonographic lesions to those distinguishing between PsA and controls. Of the 8 sonographic elementary lesions (hypoechogenicity, thickening, enthesophyte, calcification, erosion, bone proliferation, Doppler signal, and bursitis), we identified the following 5 lesions as distinguishing between PsA and controls: hypoechogenicity, thickening, enthesophyte, erosions, and Doppler signal. The summary of the results of regression models is shown in Table 3.

Entheseal sites distinguishing PsA from controls. In the next step, we aimed to narrow down the number of entheseal sites by identifying the optimal combination of entheseal sites that distinguishes PsA from controls. We first fitted 11 logistic regression models for each of the entheseal sites using the 5 elementary lesions identified in the previous stage (hypoechogenicity, thickening, enthesophyte, erosions, and Doppler signal). The regression coefficients from each of the 11 sites were then included in a single regression model as covariates (predictors) for PsA. The presence of Doppler signal and enthe-

Table 3. Summary of the elementary lesions significantly associated with PsA versus controls by entheseal site in logistic regression analysis.

\begin{tabular}{|c|c|c|c|c|c|c|c|c|c|c|c|}
\hline Variables & QT & DP & $\mathrm{TT}$ & AT & $\mathrm{PF}$ & $\mathrm{TC}$ & LE & ME & $\mathrm{TP}$ & DT & SS \\
\hline Structure & & & & & & $\mathrm{X}$ & $\mathrm{X}$ & & & & \\
\hline Thickening & & $\mathrm{X}$ & $\mathrm{X}$ & & $\mathrm{X}$ & & & & & & \\
\hline Enthesophyte any type (grade $>0$ ) & $\mathrm{X}$ & $\mathrm{X}$ & & & & & $\mathrm{X}$ & $\mathrm{X}$ & & & \\
\hline Enthesophyte any type (grade $>1$ ) & & $\mathrm{X}$ & & & & & & & & & \\
\hline Enthesophyte any type (grade $>2$ ) & & $\mathrm{X}$ & & & & & & & & & \\
\hline Calcifications & & & & & & & & & & & \\
\hline Erosions & & & & $\mathrm{X}$ & & & $\mathrm{X}$ & & $\mathrm{X}$ & & $\mathrm{X}$ \\
\hline \multicolumn{12}{|l|}{ Bone irregularities } \\
\hline Doppler $($ grade $>0)$ & & & $X$ & & & $\mathrm{X}$ & & & & & \\
\hline \multicolumn{12}{|l|}{ Doppler $($ grade $>1)$} \\
\hline \multicolumn{12}{|l|}{ Doppler (grade > 2) } \\
\hline
\end{tabular}

$\mathrm{X}$ marks elementary lesions that were associated with PsA by entheseal site in logistic regression analysis at $\mathrm{p}<0.10$. PsA: psoriatic arthritis; AT: Achilles tendon; DT: deltoid insertion; DP: patellar ligament insertion into distal patella; LE: common extensor tendon insertion into lateral epicondyle; ME: common flexor tendon insertion into medial epicondyle; PF: plantar fascia; QT: quadriceps tendon; SS: supraspinatus; TC: triceps tendon; TP: tibialis posterior; TT: patellar ligament insertion into tibial tuberosity. 
sophytes was initially analyzed as binary variables (yes/no, Model A) and subsequently as continuous variables by severity (grade 0-3, Model B). The results of the 2 logistic regression models showing the optimal combination of entheseal sites that distinguished between PsA and controls are shown in Table 4 .

The final reduced logistic regression analysis using binary variables (Model A) included the following 6 entheseal sites: patellar ligament insertions into distal patella and tibial tuberosity, Achilles tendon, plantar fascia, lateral epicondyle, and supraspinatus. The AUC for this model was 0.93 (95\% CI 0.88-0.98, Figure 1). Using Doppler and enthesophytes as continuous variables (Model B) led to similar results with the same 6 entheseal sites remaining in the model. The AUC of this model was 0.94 (95\% CI 0.89-0.99, Figure 1).

\section{DISCUSSION}

Our study provides important preliminary information about the performance of the various sonographic entheseal lesions and sites in PsA. We identified 5 elementary lesions and 6 entheseal sites in the lower and upper extremities that effectively distinguished between patients with PsA and age- and sex-matched controls. Our results will inform the development of a novel sonographic enthesitis score for PsA.

A global sonographic enthesitis score is ideally used for multiple purposes such as diagnosis of the disease, distinguishing between disease states (remission vs active disease), and monitoring of treatment response. However, the concept of "sonographic enthesitis" is complex and comprises a mix of elementary lesions that represent actively inflamed enthe-

Table 4. Logistic regression analysis for the entheseal sites that were associated with PsA versus controls.

\begin{tabular}{|c|c|c|c|c|}
\hline \multirow[t]{2}{*}{ Variables } & \multicolumn{2}{|c|}{ Multivariable Reduced Model A* } & \multicolumn{2}{|c|}{ Multivariable Reduced Model B** } \\
\hline & Estimate (SE) & $\mathrm{p}$ & Estimate (SE) & $\mathrm{p}$ \\
\hline Intercept & $0.50(0.43)$ & & $0.53(0.44)$ & \\
\hline Patellar ligament insertion into tibial tuberosity & $0.69(0.38)$ & 0.07 & $0.81(0.44)$ & 0.06 \\
\hline Achilles tendon & $1.07(0.58)$ & 0.06 & $0.95(0.53)$ & 0.07 \\
\hline Plantar fascia & $0.93(0.49)$ & 0.06 & $0.89(0.48)$ & 0.06 \\
\hline
\end{tabular}

* All 5 elementary lesions were scored as binary variables $(0=$ absent, $1=$ present $)$.** Doppler signal and enthesophytes were scored by severity on a scale of $0-3$. The remaining variables were scored as $0=$ absent, $1=$ present. PsA: psoriatic arthritis.

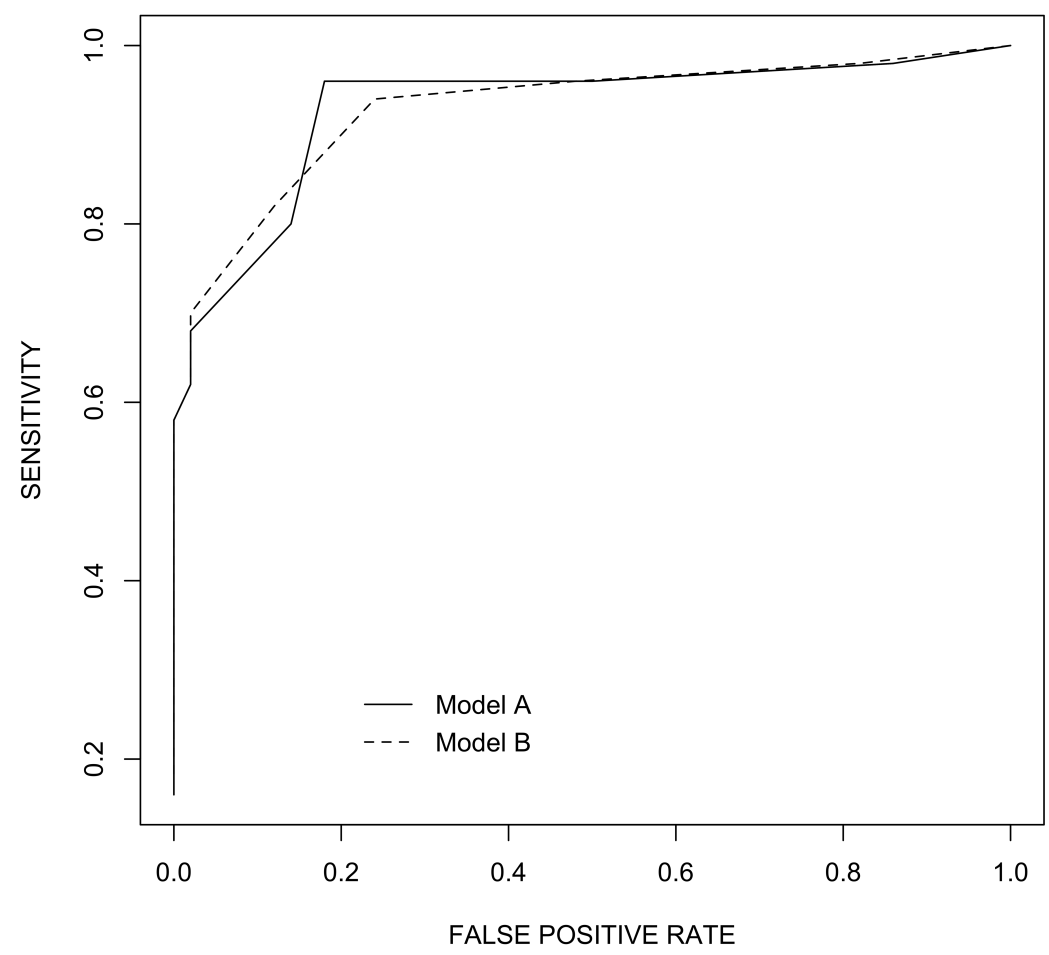

Figure 1. Receiver-operating curves for the final regression models. Model A: continuous line; Model B: dotted line. 
sitis (e.g., Doppler vascularization) and irreversible entheseal damage (e.g., bone erosion, enthesophyte). Therefore, we decided to focus on identifying the sonographic entheseal lesions and sites that can be used to distinguish patients with PsA from nonpsoriatic individuals of similar age and sex. This is important because sonographic entheseal abnormalities may also be found in individuals without inflammatory diseases secondary to age-related degenerative changes and biomechanical stress, and entheseal lesions can be influenced by age, BMI, and sex ${ }^{14,15}$. This study was the first step toward identifying lesions that can distinguish PsA from nonpsoriatic individuals. It will inform future research to assess the discrimination capacity of sonographic enthesitis (e.g., PsA from patients with cutaneous psoriasis alone), as well as in therapeutic trials to investigate response of inflammatory lesions to disease management.

In general, the frequency of each individual elementary lesion was relatively low, leading to a relatively low sensitivity to distinguish PsA from controls when considering each elementary lesion separately. Additionally, the pattern of distribution of the sonographic elementary lesions varied widely across the 11 entheseal sites assessed. This has led to differences in predictive ability of elementary lesions depending on the specific entheseal sites and suggested that a global score for enthesitis should include several elementary lesions and entheseal sites. The regression analysis revealed that using 5 sonographic elementary lesions assessed in 6 entheseal sites in the upper and lower extremities, we could distinguish between patients with PsA and controls with a high degree of confidence. It should also be noted that all the elementary lesions identified in our study as being important including enthesophytes, erosions, thickened entheseal insertion, and power Doppler signals have been previously noted by other enthesitis studies involving PsA and SpA patients ${ }^{9}$ and are included in the OMERACT definition for sonographic entheseal lesions ${ }^{14}$.

One of the primary aims of our study was to narrow down the number of entheseal sites included in a new sonographic enthesitis score using a data-driven approach. We have reduced the number of entheseal sites from 14 ( 28 bilaterally) to 6 (12 bilaterally) sites in a multistep process. The identified sites in the lower limbs are included in other sonographic or clinical enthesitis scores ${ }^{16}$. In addition, we identified 2 entheseal sites in the upper limbs that had additional predictive value, including the supraspinatus attachment to the greater tubercle of the humerus and the common extensor tendon attachment to the lateral epicondyle. Some, but not all, of the 6 identified sites are reflected in clinical enthesitis scores. We found that some of the sites included in these clinical scoring systems can be challenging to evaluate in US or did not contribute to prediction. By determining the technical aspects of various entheseal sites, this information contributes to focusing on specific entheseal sites to further examine for future studies regarding patients with PsA or cutaneous psoriasis.

Our study has some limitations. First, it did not assess the interrater reliability of acquisition of images. However, we have found high intra- and interrater reliability in scoring the different entheseal lesions. Second, no information was collected about sports activity that may confound entheseal changes; however, we did account for other confounders such as age, sex, and BMI. Finally, the study included a relatively small selected sample of patients that may not represent the global population of patients with PsA. As an exploratory study, we have chosen to examine patients with early and established disease, to record the entire spectrum of entheseal changes for greater applicability. Our focus was to identify significant elementary lesions rather than the effect of treatment options on PsA. This new scoring system will need to be further evaluated in expanded study populations to test its ability to distinguish between PsA and controls, as well as future application to distinguish patients with PsA from those with cutaneous psoriasis. An assessment of the discriminative ability of the derived scoring system in an independent validation sample is an important next step because the data in the small sample were overfitted in these analyses.

Our study contributes to the application of US for patients with PsA by identifying elemental sonographic abnormalities that distinguish PsA from controls. The use of a new sonographic score for enthesitis assessment contributes to new understanding of PsA and introduces a clinically relevant use of musculoskeletal US at the bedside.

\section{ONLINE SUPPLEMENT}

Supplementary material accompanies the online version of this article.

\section{REFERENCES}

1. Polachek A, Li S, Chandran V, Gladman D. Clinical enthesitis in a prospective longitudinal psoriatic arthritis cohort: Incidence, prevalence, characteristics and outcome. Arthritis Care Res 2017;69;1685-91.

2. Polachek A, Cook R, Chandran V, Gladman DD, Eder L. The association between sonographic enthesitis and radiographic damage in psoriatic arthritis. Arthritis Res Ther 2017;19:189.

3. McGonagle D, Gibbon W, Emery P. Classification of inflammatory arthritis by enthesitis. Lancet 1998;352:1137-40.

4. McGonagle DG, Helliwell P, Veale D. Enthesitis in psoriatic disease. Dermatology 2012;225:100-9.

5. Coates LC, Kavanaugh A, Mease PJ, Soriano ER, Laura Acosta-Felquer M, Armstrong AW, et al. Group for Research and Assessment of Psoriasis and Psoriatic Arthritis 2015 treatment recommendations for psoriatic arthritis. Arthritis Rheumatol 2016;68:1060-71.

6. Kristensen S, Christensen JH, Schmidt EB, Olesen JL, Johansen $\mathrm{MB}$, Arvesen KB, et al. Assessment of enthesitis in patients with psoriatic arthritis using clinical examination and ultrasound. Muscles Ligaments Tendons J 2016;6:241-7.

7. Balint PV, Kane D, Wilson H, McInnes IB, Sturrock RD. Ultrasonography of entheseal insertions in the lower limb in spondyloarthropathy. Ann Rheum Dis 2002;61:905-10.

8. Freeston JE, Coates LC, Nam JL, Moverley AR, Hensor EM,

Personal non-commercial use only. The Journal of Rheumatology Copyright (C) 2019. All rights reserved. 
Wakefield RJ, et al. Is there subclinical synovitis in early psoriatic arthritis? A clinical comparison with gray-scale and power Doppler ultrasound. Arthritis Care Res 2014;66:432-9.

9. Zabotti A, Bandinelli F, Batticciotto A, Scire CA, Iagnocco A, Sakellariou G, et al; Musculoskeletal Ultrasound Study Group of the Italian Society of Rheumatology. Musculoskeletal ultrasonography for psoriatic arthritis and psoriasis patients: a systematic literature review. Rheumatology 2017;56:1518-32.

10. de Miguel E, Munoz-Fernandez S, Castillo C, Cobo-Ibanez T, Martin-Mola E. Diagnostic accuracy of enthesis ultrasound in the diagnosis of early spondyloarthritis. Ann Rheum Dis 2011;70:434-9.

11. Terslev L, Naredo E, Iagnocco A, Balint PV, Wakefield RJ, Aegerter $\mathrm{P}$, et al; Outcome Measures in Rheumatology Ultrasound Task Force. Defining enthesitis in spondyloarthritis by ultrasound: results of a Delphi process and of a reliability reading exercise. Arthritis Care Res 2014;66:741-8

12. Elalouf O, Bakirci Ureyen S, Touma Z, Anderson M, Kaeley G, Aydin SZ, et al. Psoriatic arthritis sonographic enthesitis instruments: a systematic review of the literature. J Rheumatol 2018 Jul 15 (E-pub ahead of print).

13. Taylor W, Gladman D, Helliwell P, Marchesoni A, Mease P, Mielants H, et al; CASPAR Study Group. Classification criteria for psoriatic arthritis: development of new criteria from a large international study. Arthritis Rheumatol 2006;54:2665-73.

14. Lanfranchi MA, Leluc O, Tavano A, Wormser C, Morange S, Chagnaud $\mathrm{C}$, et al. Are ultrasound findings similar in patients with axial spondyloarthritis and in athlete entheses? J Rheumatol 2017;44:609-12.

15. Eder L, Jayakar J, Thavaneswaran A, Haddad A, Chandran V, Salonen D, et al. Is the MAdrid Sonographic Enthesitis Index useful for differentiating psoriatic arthritis from psoriasis alone and healthy controls? J Rheumatol 2014;41:466-72.

16. Coates L. Outcome Measures in Psoriatic Arthritis. Rheum Dis Clin North Am 2015;41:699-710. 


\section{Correction}

The Role of Ultrasound in Psoriatic Arthritis - Do We Need a Score? [editorial]

D'Agostino MA, Coates LC. The role of ultrasound in psoriatic arthritis - do we need a score? J Rheumatol 2019; doi:10.3899/jrheum.181044. Reference 20 of this editorial has incorrect citation numbers. The numbers should be 2019;46:384-90.

doi:10.3899/jrheum.181044.C1 